\author{
United Nations Educational Scientific and Cultural Organization \\ and \\ International Atomic Energy Agency
}

THE ABDUS SALAM INTERNATIONAL CENTRE FOR THEORETICAL PHYSICS

\title{
GYROGROUPS AND HOMOGENEOUS LOOPS
}

\author{
A. Nourou Issa ${ }^{1}$ \\ Institut de Mathématiques et de Sciences Physiques, Université Nationale du Bénin, \\ B.P. 613 Porto-Novo, Bénin ${ }^{2}$ \\ and \\ The Abdus Salam International Centre for Theoretical Physics, Trieste, Italy.
}

\begin{abstract}
We stress on the basic role of homogeneous loops in the loop-theoretical interpretations of gyrogroups. We point out that the (left) Bruck identity always occurs in smooth homogeneous loops with automorphic inverse property and that gyrogroups can be viewed as homogeneous loops with one or two identities.
\end{abstract}

MIRAMARE - TRIESTE

June 1998

\footnotetext{
${ }^{1}$ Regular Associate of the Abdus Salam ICTP.

${ }^{2}$ Permanent address. Fax: (229) 212525
} 


\section{Introduction.}

In order to give a suitable interpretation of the strange phenomenon that is observed in the composition of the relativistically admissible velocities, Abraham A. Ungar introduced the concept of a gyrogroup $([19,20,21])$. It happens that gyrogroups are noncommutative and nonassociative algebraic structures and this noncommutativity-nonassociativity turns out to be generated by the Thomas precession, well-known in the special theory of relativity. Gyrogroups also revealed themselves to be specially fitting in order to deal with formerly unsolved problems in special relativity (e.g. the problem of determining the Lorentz transformation that links given initial and final time-like 4-vectors). Further (see [22]) gyrogroups were split up into gyrocommutative gyrogroups and nongyrocommutative ones just as groups split up into abelian and nonabelian (we notice that the prior concept of a gyrogroup in $[19,20,21]$ is relevant to the one of a gyrocommutative gyrogroup).

It turns out that introducing (gyrocommutative) gyrogroups, Ungar gave a concrete physical realization to formerly well-known algebraic systems called $K$-loops. The $K$-loops were discovered in a purely mathematical context by Karzel H. $([6,7])$ in his study of neardomains (the additive structure of a neardomain is precisely the $K$-loop). The view of a (gyrocommutative) gyrogroup as a $K$-loop rose the interest to both of these objects (see $[18,17,10,9]$ ).

Although the algebraic interpretations of gyrogroups heavily lay on the structure of homogeneous loops, the basic role of such loops is somewhat overlooked although a way in this direction is shown in [10]. In this paper we wish to emphasize a little more on this role. The major motivation for that is the fact that a gyrogroup (nongyrocommutative or gyrocommutative) can be viewed as a homogeneous loop with one or two identities according to whether that gyrogroup is nongyrocommutative or gyrocommutative and that the (left) Bruck identity always holds in a smooth homogeneous loop with automorphic inverse property (we get the same result if we replace the smoothness by a suitable identity; see sections 4 and 5). Indeed the (left) Bruck identity (which corresponds to the gyrocommutative law in gyrogroups) is a particular case of a more general identity characterizing homogeneous loops with automorphic inverse property (we call it the Kikkawa identity). So that the gyrocommutative law is a particular case of the Kikkawa identity. A hint at hyporeductive loops is done: diassociative (gyrocommutative) gyrogroups are Moufang A-loops and therefore constitute a type of (left) hyporeductive loops (section 4). The section 3 has a survey character since therein we give a brief account on the axiomatic approach to gyrogroups and their successive loop-theoretical views. In section 2 we display the useful notions from loop theory. We conclude in section 6 with some observations.

\section{Background and notations.}

Here we record some notions in loop theory that will be for later use. For more information on the general theory of loops one may refer to $[1,2,13]$.

A loop $(Q, ., e)$ is a set $Q$ together with a binary operation (.), i.e. a groupoid, such that each of the equations $a . x=b$ and $y . a=b$ has a unique solution for any $a, b$ in $Q$ and such that there exists a two-sided identity element $e$. The loop $(Q, ., e)$ is said to be $s m o o t h$ whenever $Q$ 
is a smooth (or differentiable) manifold and the loop multiplication (.) is smooth. The (left or right) inverse of an element $a \in Q$ is denoted by $a^{-1}$. Roughly a loop could be seen as a "group that is nonassociative".

The left permutation $L_{a}$ and the right permutation $R_{a}$ of $(Q, ., e)$ are defined as $L_{a} x=a . x$ and $R_{a} x=x . a$. A permutation $\alpha$ of $Q$ such that $\alpha e=e$ is called an inner mapping of $Q$. The set of all inner mappings of a given loop $Q$ constitutes a group usually denoted by $I(Q)$. In what follows we will be interested in a special kind of inner mappings, namely the left inner mappings $l_{a, b}$ defined by $l_{a, b}=L_{a . b}^{-1} \circ L_{a} \circ L_{b}$ or, what is equivalent, $a b . l_{a, b} c=a . b c$ so that $l_{a, b}$ may be viewed as a "mapping correction" up to associativity in loops.

A loop $Q$ is called a left $A$-loop if the left inner mappings $l_{a, b}, a, b \in Q$, are automorphisms of $Q$, that is if the following identity holds:

$$
l_{a, b} \circ L_{x} \circ l_{a, b}^{-1}=L_{l_{a, b} x} \quad(\text { left } A-\text { identity }) .
$$

A homogeneous loop is a left $A$-loop with the left inverse property $L_{a}^{-1}=L_{a^{-1}}$.

The concept of a left $A$-loop and of a homogeneous loop was introduced by Kikkawa M. in [8]. Throughout this paper we shall deal mainly with homogeneous loops.

A loop $Q$ is said to have the automorphic inverse property (Kikkawa called it the symmetric property) if the inverse mapping $J(a)=a^{-1}$ is an automorphism of $Q$, that is if the identity

$$
(a b)^{-1}=a^{-1} \cdot b^{-1} \quad \text { (automorphic inverse property) }
$$

holds in $Q$.

Beside $A$-loops one of the most interesting class of loops is the one of Bol loops. A loop $Q$ is called a left Bol loop if the identity

$$
(a . b a) c=a(b . a c) \quad(\text { left Bol identity) }
$$

holds in $Q$. If, in addition, $Q$ has the automorphic inverse property then $Q$ is called a (left) Bruck loop. The identity

$$
a b . a b=a(b . b a)
$$

in Bol loops is called the (left) Bruck identity. For more information on the algebraic theory of Bol and Bruck loops one may refer, e.g., to Robinson D.A. [14] and Goodaire E.G. [4]. The relationship between left $\mathrm{Bol}$ loops and left $A$-loops were established in a number of papers (see e.g. $[3,15])$. We have the following

LEMMA 1. ([5], lemma 1 and [9], lemma 2.5). A loop $Q$ satisfies the left Bol identity if, and only if,

$$
l_{b, a b} \circ l_{a, b}=i d
$$

for any $a, b \in Q$.

Remark. From the proof of lemma 1 of [5], it could be seen that the equivalence between (5) and the left Bol identity is true for any loop without the requirement of the left $A$-identity in the considered loop. In [9] Kreuzer A. showed this equivalence straightforwardly (see also 
[10]). Besides, somewhat anticipating, we observe that the identity (5) is a key link between (left) $A$-loops, Bol loops and $K$-loops (section 4 ).

We recall the notion of a $K$-loop $([7,10,9])$.

A loop $Q$ is called a (left) $K$-loop if it is a left $A$-loop with the automorphic inverse property and satisfies the following identity

$$
l_{a, b}=l_{a, b a}
$$

for any $a, b \in Q$. As it could be seen from [20,21], the property (6) for $K$-loops turns out to play a fundamental role in the purely physical concept of gyrogroups and their applications.

\section{Gyrogroups, K-loops and Bruck loops.}

In this section we give a brief review on gyrogroups $([19,20,21])$ and their loop-theoretical structures $([7,9,10,17,18])$.

Ungar A.A. discovered ([19]) that the set of all 3-dimensional relativistically admissible velocities, with their relativistic addition law, constitute a noncommutative and nonassociative groupoid. Such an algebraic structure satisfies some specific identities involving the Thomas precession. From the special theory of relativity it is known that it is due to the presence of the Thomas precession that proper Lorentz transformations (called also boosts) do not constitute a group (at this point we recall that the nonassociativity of the boosts product has been previously pointed out by a number of physicists (see, e.g., [12])). Further, Ungar illustrated the ability of the above mentioned algebraic structure in solving some standing physical problems. Next, in the light of the works of Karzel H. ([6, 7]), Ungar gave an axiomatic approach to that algebraic structure (see, e.g., [20,21]) and called it a gyrogroup. Precisely, such an axiomatic approach is of most interest from a loop-theoretical point of view.

Let $V_{c}$ denote the set of relativistically admissible velocities, that is $V_{c}$ is the set of abstract velocities $v$ such that $|v|<c$, where $|v|=\sqrt{(v, v)}>0$ (here $(-,-)$ denotes the scalar product in the 3-dimensional Euclidian space) and $c$ is an arbitrarily fixed positive constant (usually it is the speed of light in empty space). Define on $V_{c}$ a relativistic velocity addition $\oplus$ as follows:

$$
u \oplus v=\left(1+(u, v) / c^{2}\right)^{-1 / 2}\left[u+\gamma_{u}^{-1} v+c^{-2} \gamma_{u}\left(\gamma_{u}+1\right)^{-1}(u \times(u \times v))\right]
$$

where $\gamma_{u}=\left(1-(|u| / c)^{2}\right)^{-1 / 2}$ and $u \times v$ is the vector product of velocities $u$ and $v$. The system $\left(V_{c}, \oplus\right)$ turns out to be a noncommutative and nonassociative groupoid. As mentioned above, the nonassociativity of $\left(V_{c}, \oplus\right)$ arises from the presence of the Thomas precession (we will carry the notation $g y r_{u, v}$ to denote the Thomas precession generated by $u, v \in V_{c}$ ). Ungar established $([19,20,21])$ several properties linking the composition law $\oplus$ and the Thomas precession. Some of those properties are listed here:

$$
\begin{gathered}
u \oplus(v \oplus w)=(u \oplus v) \oplus g y r_{u, v} w \quad \text { (left weak associative law) } \\
(u \oplus v) \oplus w=u \oplus\left(v \oplus g y r_{v, u} w\right) \text { (right weak associative law) } \\
0 \oplus u=u \oplus 0=0 \text { (existence of identity) } \\
(-u) \oplus u=u \oplus(-u)=0 \text { (existence of inverse) }
\end{gathered}
$$




$$
(u \oplus v)=g y r_{u, v}(v \oplus u) .(\text { weak commutative law })
$$

Moreover the Thomas precession $g y r_{u, v}$ possesses its peculiar properties:

$$
\begin{array}{r}
g y r_{0, v}=i d \text { (identity property) } \\
g y r_{u \oplus v, v}=g y r_{u, v}=g y r_{u, v \oplus u}(\text { loop property) } \\
g y r_{u, v}^{-1}=g y r_{v, u} \\
g y r_{-u,-v}=g y r_{u, v} \\
g y r_{u,-v}=g y r_{u \oplus u,-(u \oplus v)} \circ g y r_{u, v} \\
g y r_{u, v \oplus w}=g y r_{u \oplus v, g y r_{u, v} w} \circ g y r_{u, v} \circ g y r_{w, v} \\
g y r_{u, v}=g y r_{-g y r_{u, v} v, u} .
\end{array}
$$

Further (see [22]) the loop property (14) splits up into the left loop property $g y r_{u \oplus v, v}=g y r_{u, v}$ and the right loop property $g y r_{u, v \oplus u}=g y r_{u, v}$. The weak commutative law and the loop properties were found to play a central role in the loop-theoretical interpretations of the groupoid $\left(V_{c}, \oplus\right)$. Further, the "gyroterminology" ([21]) were introduced in order to accomodate the peculiar features of the composition of relativistically admissible velocities due to the Thomas precession. Since the precession characterises the gyration of the rotation axis of a rotating frame, the Thomas precession was called the Thomas gyration. Likewise the right (left) weak associative law, the weak commutative law and the identity property were respectively called the right (left) gyroassociative law, the gyrocommutative law and the identity gyroautomorphism. We note that the right (left) gyroassociative law was called in [21] the left (right) gyroassociative law.

A groupoid satisfying (8)-(11),(13) is called a weakly associative groupoid and the one satisfying, in addition, the identity (14) is called a (nongyrocommutative) gyrogroup. A nongyrocommutative gyrogroup satisfying (12) is called a gyrocommutative gyrogroup ([22]).

It was noticed that the groupoid $\left(V_{c}, \oplus\right)$ actually forms a loop ([21]) with the null velocity 0 as its identity and the $g^{y r_{u, v}}$ (for any $u, v \in V_{c}$ ) are automorphisms (the so-called gyroautomorphisms $)$ of the loop $\left(V_{c}, \oplus, 0\right)$ and also that $g y r_{u, v}$ are, in fact, the well-known left inner mappings $l_{u, v}$ of loop theory $([17])$. Here we observe that from the standpoint of loop theory it seems more suitable to view the left gyroassociative law (8) as a definition of $g y r_{u, v}$ rather than the expression $g y r_{u, v} w=-(u \oplus v) \oplus(u \oplus(v \oplus w))$ which is a consequence of the left inverse property that occurs in gyrogroups (see [17]).

It turns out that the concept of a (gyrocommutative) gyrogroup adequately reflects the one of a formerly well-known mathematical object, namely the $K$-loop, introduced by Karzel H. ([6, $7]$ ) in his study of neardomains (for more information on $K$-loops one may refer, e.g., to [23, 10,9]). Wefelscheid H. and Kreuzer A. established in [10] that any $K$-loop is a left Bol loop with the automorphic inverse property, i.e. a Bruck loop. Recently ([9]) Kreuzer A. proved that $K$-loops and Bruck loops are actually the same. At this point we mention a paper by Sabinin L.V. and Mikheev P.O. ([18]), where, analyzing a composition law of relativistically admissible velocities, they established several properties of that law particularly the left Bol identity and 
the automorphic inverse property. Further, Sabinin L.V. ([17]) showed straightforwardly that a (gyrocommutative) gyrogroup is a Bruck loop.

We may sum up the observations above in the following

THEOREM 1. ([17,9]). A gyrocommutative gyrogroup is a left Bruck loop.

The occurrence of the left Bruck identity in gyrocommutative gyrogroups will be extended in section 4 to the general case of homogeneous loops with automorphic inverse property.

\section{Gyrocommutative gyrogroups and homogeneous loops.}

Here we draw out some specific properties of homogeneous loops with automorphic inverse property. It happens that these properties are also characteristic properties of gyrocommutative gyrogroups and therefore we establish that all axioms defining gyrocommutative gyrogroups are properties of homogeneous loops with automorphic inverse property satisfying the identity (5). We point out that the gyrocommutative law is a "trivialization" of a more general identity that occurs in such gyrogroups and also that the gyrosum inversion rule ([22]) follows from other axioms of gyrogroups. We observe that diassociative gyrocommutative gyrogroups are (left) hyporeductive loops (they are actually Moufang A-loops) but unfortunately we wonder whether there exist such gyrogroups and what is their physical meaning.

LEMMA 2. Let $G$ be a homogeneous loop. Then $G$ has the automorphic inverse property if, and only if,

$$
L_{x y} \circ L_{x y}=L_{x} \circ L_{y} \circ L_{y} \circ L_{x}
$$

for any $x, y$ in $G$. Moreover, if $G$ has the automorphic inverse property, then

$$
l_{x, y}=l_{x^{-1}, y^{-1}}
$$

Proof. (see [8], Proposition 1.13).

We will refer to (20) as the Kikkawa identity.

COROLLARY 1. The left Bruck identity (4) holds in every smooth homogeneous loop with automorphic inverse property.

Proof. Applying the Kikkawa identity (20) to the identity element we get the left Bruck identity since any smooth homogeneous loop with the automorphic inverse property has the left alternative property ([8], lemma 6.2 ).

In view of lemma 1 , one can observe that we will get the same result as in corollary 1 above if we replace the smoothness by the identity (5). Since $K$-loops are first and foremost homogeneous loops with automorphic inverse property, we readily have 
COROLLARY 2. Every left $K$-loop satisfies the left Bruck identity.

One notices that corollary 2 also could be seen as a consequence of theorem 3.4 in [9].

LEMMA 3. For any homogeneous loop $G$ with automorphic inverse property the identity

$$
l_{x, y}=l_{y, x}^{-1} .
$$

holds for any $x, y$ in $G$.

Proof. First we observe that since $l_{x, y}$ are automorphisms of $G, l_{x, y} \circ L_{y^{-1} x^{-1}}(z)=(x y)^{-1} \cdot l_{x, y} z \quad(z \in$ $G)$, that is $l_{x, y} \circ L_{y^{-1} x^{-1}}=L_{x y}^{-1} \circ l_{x, y}$. Therefore $l_{x, y}=L_{x y} \circ l_{x, y} \circ L_{y^{-1} x^{-1}}$ and then $l_{x, y}^{-1}=$ $L_{y^{-1} x^{-1}}^{-1} \circ L_{y^{-1}} \circ L_{x^{-1}}=l_{y^{-1}, x^{-1}}=l_{y, x}$ (by $\left.(21)\right)$.

One can notice that the identity (22) reads (15) in gyroterminology.

THEOREM 2. The gyrosum inversion rule

$$
-(u \oplus v)=g y r_{u, v}((-v) \oplus(-u))
$$

is a consequence of other axioms defining a gyrogroup.

Proof. We notice first that the left inverse property occurs also in nongyrocommutative gyrogroups since it follows from the left loop property, the gyroautomorphism identity and the inverse identity. Next, by the left gyroassociative law and the left invertibility, we have gyr $_{u, v} w=$ $-(u \oplus v) \oplus(u \oplus(v \oplus w))$ and if we set $w=(-v) \oplus(-u)$ we will get

$$
\begin{aligned}
\text { gyr }_{u, v}((-v) \oplus(-u)) & =-(u \oplus v) \oplus(u \oplus(v \oplus((-v) \oplus(-u)) \\
& =-(u \oplus v) \oplus(u \oplus(-u)) \text { (by the left invertibility) } \\
& =-(u \oplus v) \oplus 0 \text { (by the inverse identity) } \\
& =-(u \oplus v) \text { (by the identity property). }
\end{aligned}
$$

LEMMA 4. If a gyrogroup satisfies the gyrocommutative law, then it has the automorphic inverse property.

Proof. We have

$$
\begin{aligned}
-(u \oplus v) & =g_{y r_{u, u}}((-v) \oplus(-u)) \text { (by the gyrosum inversion rule) } \\
& =-(u) \oplus(v) \text { (by the gyrocommutative law) }
\end{aligned}
$$

It turns out that the converse to lemma 4 is also true (see [22], theorem 5.9).

THEOREM 3. The identity

$$
(x \oplus y) \oplus z=g y r_{x, y}(y \oplus(x \oplus z))
$$


holds in any gyrocommutative gyrogroup and the gyrocommutative law is a "trivialization" of (23). Moreover any gyrocommutative gyrogroup is a homogeneous loop with automorphic inverse property satisfying (5).

Proof. Making use of the techniques developed in [8] for homogeneous loops, we can perform the following transformations: consider $-(u \oplus v)$ then we have

$-(u \oplus v)=(u \oplus v)(-(u \oplus v))^{2}=(u \oplus v) g_{y r_{u, v}}((-v) \oplus(-u))^{2}$

$=u \oplus\left(v \oplus((-v) \oplus(-u))^{2}\right)$. Thus

$$
-(u \oplus v)=u \oplus\left(v \oplus((-v) \oplus(-u))^{2} .\right.
$$

Replace $u$ by $-u$ in (24) and then, using the automorphic inverse property (see lemma 4), we get $u \oplus(-v)=(-u) \oplus\left(v \oplus((-v) \oplus u)^{2}\right)$, that is

$$
u \oplus(u \oplus(-v))=v \oplus((-v) \oplus u)^{2}
$$

and then substituting $w \oplus u$ for $u$ and $w \oplus v$ for $v$, we get

$$
(w \oplus u) \oplus((w \oplus u) \oplus(-(w \oplus v)))=(w \oplus v) \oplus((-(w \oplus v)) \oplus(w \oplus u))^{2} .
$$

Now we can transform $(-(w \oplus v) \oplus(w \oplus u))^{2}$ using the fact that $-(w \oplus v) \oplus(w \oplus u)=g y r_{w, v}((-v) \oplus$ $u)$ and that $g y r_{w, v}$ is an automorphism. We have $\left((-(w \oplus v) \oplus(w \oplus u))^{2}=g y r_{w, v}\left(((-v) \oplus u)^{2}\right)\right.$ $=(-(w \oplus v)) \oplus\left(w \oplus\left(v \oplus((-v) \oplus u)^{2}\right)\right)$. Thus by (25) and (26), we get

$$
\begin{aligned}
(w \oplus u) \oplus((w \oplus u) \oplus(-(w \oplus v))) & =(w \oplus v) \oplus((-(w \oplus v)) \oplus(w \oplus u))^{2} \\
& =w \oplus(u \oplus(u \oplus(-v))) .
\end{aligned}
$$

Replacing $-v$ by $v$ in (27) and again using the automorphic inverse property, we get

$$
w \oplus(u \oplus(u \oplus v))=(w \oplus u) \oplus((w \oplus u) \oplus((-w) \oplus v))
$$

that is, setting $t=(-w) \oplus v$,

$$
(w \oplus u) \oplus((w \oplus u) \oplus t=w \oplus(u \oplus(u \oplus(w \oplus t)))
$$

or, finally,

$$
(w \oplus u) \oplus t=g y r_{w, u}(u \oplus(w \oplus t))
$$

so that we get (23). Besides, setting $t=0$, we obtain the gyrocommutative law.

From the axioms defining gyrocommutative gyrogroups and the results above with regard to homogeneous loops with automorphic inverse property it clearly follows that such gyrogroups are such homogeneous loops with the additional identity (5) since the right loop property, which is equivalent to the left loop property ([20]), implies the identity (5). This completes our proof.

Remark. The expression "trivialization" is used to stress on the fact that the gyrocommutative law is obtained by applying the "generalized" gyrocommutative law (23) to the identity element 0 . One easily checks up that (23) is precisely the Kikkawa identity (20) written in gyroterminology. From (23) one can draw other identities for gyrogroups that could be of interest 
for applications: for instance, replacing $z$ by $-(x \oplus(-z))$ in $(23)$ and using the automorphic inverse property and the left invertibility, one gets

$$
(x \oplus y) \oplus((-x) \oplus z)=g y r_{x, y}(y \oplus z) .
$$

The identity (29), written in loop notations, reads $x y \cdot x^{-1} z=l_{x, y}(y z)$ which is shown to be equivalent to (20) (see [8], Proposition 1.13).

THEOREM 4. The right gyroassociative law in gyrocommutative gyrogroups derives from properties of homogeneous loops with automorphic inverse property.

Proof. By definition we have $l_{u, v} w=L_{u, v}^{-1} \circ L_{u} \circ L_{v}(w)$, that is $(u \oplus v) \oplus g y r_{u, v} w=u \oplus(v \oplus w)$ (left gyroassociative law). The identity (22) holds in any homogeneous loop with automorphic inverse property or, what is equivalent, (15) holds in any gyrocommutative gyrogroup. Now in the left gyroassociative law, replace $w$ by $g y r_{v, u} z$ and it reads then

$(u \oplus v) \oplus g y r_{u, v}\left(g y r_{v, u} z\right)=u \oplus\left(v \oplus g y r_{v, u} z\right)$, that is

$$
(u \oplus v) \oplus z=u \oplus\left(v \oplus g y r_{v, u} z\right)
$$

and this is the right gyroassociative law.

THEOREM 5. Any homogeneous loop with automorphic inverse property that satisfies the identity (5) can be endowed with a gyrocommutative gyrogroup structure (in the sense of the axiomatic approach).

Proof. One needs to postulate the left inner mappings as Thomas gyrations and then, by the definition of a loop and by the specific properties of homogeneous loops with automorphic inverse property, one gets the axioms (8)-(12). Furthermore, written in gyroterminology, the identity (5) implies the right loop property and therefore one obtains a gyrocommutative gyrogroup.

Remark. The results above are expected in view of the identification of $K$-loops with left Bruck loops ([9]).

We link a type of gyrocommutative gyrogroups with hyporeductive loops (see definition in [16, $5]$ ).

THEOREM 6. Diassociative gyrocommutative gyrogroups are (left) hyporeductive loops.

Proof. From theorems 3 and 5 we have that any gyrocommutative gyrogroup is a left Bruck loop (see $[10,17,9]$ ). But as it was pointed out in [5, theorem 5], diassociative left Bruck loops are left hyporeductive loops (since they are Moufang $A$-loops). 


\section{Nongyrocommutative gyrogroups and homogeneous loops.}

In this section we point out that nongyrocommutative gyrogroups are homogeneous loops with the "left loop property". Beforehand we observed that such homogeneous loops share many properties with homogeneous loops with automorphic inverse property.

LEMMA 5. In any homogeneous loop the following identities hold:

$$
\begin{gathered}
l_{x, y}=l_{y, y^{-1} x^{-1}} \\
l_{x, y}^{-1}=l_{y^{-1}, x^{-1}}=l_{x y,(x y)^{-1} x}
\end{gathered}
$$

Proof. (See [8], lemma 1.8).

LEMMA 6. Let $L$ be a homogeneous loop with the identity $l_{x, y}=l_{x, y, y}$ (i.e. the left loop property). Then $L$ has the following properties:

$$
\begin{array}{r}
l_{x, y}=l_{x^{-1}, y^{-1} x^{-1}} \\
l_{x, y}=l_{x^{-1}, y^{-1}} \\
l_{x, y}=l_{y, x}^{-1} \\
l_{x, y, y}=l_{y, y^{-1} x^{-1}}
\end{array}
$$

Proof. From (30) and applying the left loop property and the left inverse property we have $l_{x, y}=l_{y, y^{-1} x^{-1}}=l_{y \cdot\left(y^{-1} x^{-1}\right), y^{-1} x^{-1}}=l_{x^{-1}, y^{-1} x^{-1}}$ so that we get (32). The first equality of (31) gives

$$
\begin{aligned}
l_{x, y}^{-1} & =l_{y^{-1}, x^{-1}} \\
& =l_{y^{-1} x^{-1}, x^{-1}} \quad \text { (by the left loop property) } \\
& =l_{x,\left(y^{-1} x^{-1}\right)^{-1}}^{-1} \quad(\text { by }(31)) .
\end{aligned}
$$

Therefore, we draw $l_{x, y}=l_{x,\left(y^{-1} x^{-1}\right)^{-1}}$ and thus, with (32), $l_{x,\left(y^{-1} x^{-1}\right)^{-1}}=l_{x^{-1}, y^{-1} x^{-1}}$ that is, replacing $y^{-1} x^{-1}$ by $z, l_{x, z^{-1}}=l_{x^{-1}, z}$ which proves (33). Next we observe that (34) follows straightforwardly from (31) and (33) and that (35) is a consequence of (30) and the left loop property.

Remark. One notices that the properties (33) and (34) are also properties of homogeneous loops with automorphic inverse property (see section 4). Other identities for homogeneous loops with automorphic inverse property could be shown to be true for homogeneous loops with the left loop property as, for example, the identity $l_{x, y z}=l_{x y, l_{x, y}} \circ l_{x, y} \circ l_{z, y}$.

LEMMA 7. In any homogeneous loop with the left loop property, the following identities hold:

$$
\begin{array}{r}
l_{x^{-1}, x y} \circ l_{x, y}=i d \\
\left.l_{y, x y} \circ l_{x, y}=i d \quad \text { (see identity }(5)\right) \\
l_{x, y}=l_{x, y x} .
\end{array}
$$


Proof. We have

$$
\begin{aligned}
l_{x^{-1}, x y} \circ l_{x, y} & =l_{y^{-1}, x^{-1}} \circ l_{x, y}(\text { by }(30)) \\
& =l_{y, x} \circ l_{x, y}(\text { by }(33)) \\
& =i d(\text { by }(34))
\end{aligned}
$$

hence we get (36). Next,

$$
\begin{aligned}
l_{y, x y} \circ l_{x, y} & =l_{y, x y} \circ l_{x y, y} \text { (by the left loop property) } \\
& =i d(\text { by }(34))
\end{aligned}
$$

which proves (37). The identity (38) follows from (37) and (34).

THEOREM 7. Any loop L with the left inverse property that satisfies the left loop property is a left Bol loop and hence satisfies (37) (or (5)). If $f$ is any automorphism of L, then $f \circ l_{x, y} \circ f^{-1}=l_{f x, f y}$ for any $x, y$ in $L$.

Proof. The left loop property gives (by the left invertibility) $l_{x, y} z=$ $(x y \cdot y)^{-1}(x y \cdot y z)$. Replace $z$ by $l_{x, y}^{-1} v$ and then $(x y \cdot y) v=(x y) \cdot\left(y \cdot l_{x, y}^{-1} v\right)$ i.e., by the left invertibility, $(x y \cdot y) v=(x y) \cdot\left(x^{-1} \cdot(x y \cdot v)\right)$. Further, replacing $y$ by $x^{-1} u$, this latter equality leads to $\left(u . x^{-1} u\right) v=u\left(x^{-1} . u v\right)$ which is the left Bol identity. The identity (37) is also satisfied by virtue of lemma 1. Finally, if $f$ is any automorphism of $L$, we can perform the following transformations (having in mind the left invertibility):

$$
\begin{array}{r}
l_{f x, f y} \circ f(z)=(f x \cdot f y)^{-1}(f x \cdot(f y \cdot f z))=(f x \cdot f y)^{-1} \cdot f(x . y z) \\
=(f x \cdot f y)^{-1} \cdot f\left(x y \cdot l_{x, y} z\right)=(f x \cdot f y)^{-1}\left[(f x \cdot f y) \cdot f\left(l_{x, y} z\right)\right]=f \circ l_{x, y}(z)
\end{array}
$$

and this completes our proof.

We can now state the main result of this section.

THEOREM 8. Any nongyrocommutative gyrogroup is a homogeneous loop with the left loop property.

Proof. As noticed above the left inverse property in gyrogroups is deduced from other axioms. The left gyroassociative law implies that the gyrations $g y r_{x, y}$ are the inner mappings $l_{x, y}$. Next, since $g y r_{x, y}$ are automorphisms we then get that (nongyrocommutative) gyrogroups are homogeneous loops. Further one checks that the fundamental properties of nongyrocommutative gyrogroups obtained in [22] are either properties (30)-(38) of homogeneous loops satisfying the left loop property, or can be inferred from one or some of those properties.

Remark. By virtue of lemma 7 and lemma 1, the class of homogeneous loops with the left loop property, and hence the one of the nongyrocommutative gyrogroups viewed as loops, lies in the class of homogeneous loops that are left Bol loops. From Ungar's results in [22] and 
the results above regarding homogeneous loops with the left loop property, it follows that the counterpart of theorem 5 for the case of nongyrocommutative gyrogroups is also true.

\section{Conclusion.}

One of the most useful and interesting properties of the Thomas gyration is the loop property (left or right). This is particularly obvious in the search of Lorentz transformations linking initial and final 4-vectors ([21]). This property has also a special significance in loop theory: it is precisely due to the loop property that a weakly associative groupoid constitutes a loop and it is also one of the properties characterizing $K$-loops. If in the special theory of relativity the Thomas gyration is understood as the "rotation correction" in the passage from the nonrelativistic regime to the relativistic one, likewise in group theory the mappings $l_{x, y}$ "measures" the nonassociativity of a given groupoid. So that the identification of the Thomas gyration $g y r_{x, y}$ with the mapping $l_{x, y}$ reveals one of the most obvious concrete physical and geometrical meanings of the "nonassociativity scale" $l_{x, y}$ of loop theory. From this point of view the Ungar's gyrogroups show once again that the loop theory, and more generally the theory of nonassociative algebraic structures, more and more will be used as a powerful tool for a suitable description and explanation of some concrete physical phenomena.

It seems not exaggerating to state that one of the most suitable algebraic tools to accommodate the composition of relativistically admissible velocities is the theory of homogeneous loops (here we include $K$-loops (or Bruck loops) since these loops may be viewed as homogeneous loops with additional identities). Besides, provided the smoothness of the composition law, another tool (for gyrocommutative gyrogroups) could be the differential geometry of symmetric spaces since the structure of any smooth Bruck loop (locally) generates a structure of a symmetric space and conversely the local geodesic loop of any symmetric space has a structure of a smooth Bruck loop ([15]). It is interesting at this point to notice that applications of local geodesic loops to classical and quantum gravity are exhibited in [11].

Acknowledgements. This work was done within the framework of the Associateship Scheme of the Abdus Salam International Centre for Theoretical Physics, Trieste, Italy. Financial support from the Swedish International Development Cooperation Agency is acknowledged. I would like to thank the Abdus Salam ICTP, Trieste, for providing me with a so stimulating research environment. 


\section{References}

1. Belousov, V. D.: Foundations of the Theory of Quasigroups and Loops. Izdat. "Nauka", Moscow, 1967. (In russian)

2. Bruck, R. H.: A Survey of Binary Systems. Springer Verlag, Berlin, 1958.

3. Funk, M. and Nagy, P.T.: On collineation groups generated by Bol reflections, J. Geometry 48(1993), 63-78.

4. Goodaire, E.G. and Robinson, D.A.: Semidirect products and Bol loops, Demonstratio Mathematica 27(1994), 573-588.

5. Issa, A.N.: Examples of hypospecial loops, Algebras Groups Geom. 13(1996), 499-513.

6. Karzel, H.: Inzidenzgruppen I. Lecture Notes by Pieper, I. and Sorensen, K., University of Hamburg (1965), 123-135.

7. Karzel, H.: Zusammenhange zwischen Fastbereichen, scharf zweifach transitiven Permutationsgruppen und 2-Strukturen mit Rechtecksaxiom, Abh. Math. Sem. Univ. Hamburg 32(1968), 191-206.

8. Kikkawa, M.: Geometry of homogeneous loops, Hiroshima Math. J. 5(1975), 141-179.

9. Kreuzer, A.: Inner mappings of Bruck loops, Math. Proc. Camb. Phil. Soc. 123(1998), 53-57.

10. Kreuzer, A. and Wefelscheid, H.: On K-loops of finite order, Result Math. 25(1994), 79-102.

11. Kuusk, P. Ord, J. and Paal, E.: Geodesic multiplication and the theory of gravity, J. Math. Phys. 35(1994), 321-334.

12. Nesterov, A.I. and Stepanenko, V.A.: On methods of nonassciative algebras in geometry and physics, Preprint No.400F(1986), Institute of Physics SO AN SSSR. (In russian)

13. Pflugfelder, H. O.: Quasigroups and Loops, an Introduction. Helderman Verlag, Berlin, 1990 .

14. Robinson, D.A.: Bol loops, Trans. Amer. Math. Soc. 123(1966), 341-354.

15. Sabinin, L.V.: Methods of nonassociative algebras in differential geometry.: Supplement to the russian translation of: Kobayashi, S. and Nomizu, K., Foundations of Differential Geometry, vol.I, "Nauka", Moscow, 1981.

16. Sabinin, L.V.: Smooth hyporeductive loops, Soviet Math. Dokl. 42(1991), No.2, 524-526.

17. Sabinin, L.V.: On the gyrogroups of Ungar, Russian Math. Surveys 50(1995), No.5, 10951096.

18. Sabinin, L.V. and Mikheev, P.O.: On the law of composition of velocities in special relativity theory, Russian Math. Surveys 48(1993), No.5, 183-184.

19. Ungar, A.A.: Thomas rotation and the parametrization of the Lorentz transformation group, Foundations Phys. Lett. 1(1988), No.1, 57-89.

20. Ungar, A.A.: Weakly associative groups, Results Math. 17(1990), 149-168.

21. Ungar, A.A.: Thomas precession and its associated grouplike structure, Amer. J. Phys. $\mathbf{5 9 ( 1 9 9 1 ) , ~ N o . 9 , ~ 8 2 4 - 8 3 4 . ~}$

22. Ungar, A.A.: Thomas precession: its underlying gyrogroup axioms and their use in hyperbolic geometry and relativistic physics, Foundations Phys. 27(1997), No.6, 881-951.

23. Wefelscheid, H.: ZT-subgroups of sharply 3-transitive groups, Proc. Edinburgh Math. Soc. 23(1980), 9-14. 